\title{
Las Relaciones Internacionales desde los feminismos descoloniales. Una propuesta dialógica hacia una economía feminista descolonial
}

\author{
Selena Pizarro Gómez
}

\begin{abstract}
RESUMEN
A partir de la mitad del siglo $X X$ se consolidó el sistema capitalista moderno/colonial, por medio de la lógica eurocéntrica que agravó la brecha Norte-Sur.Así se forjaron las relaciones económicas internacionales que impusieron la engeneración y la racialización del trabajo. En este sentido, la garantía de los derechos socioeconómicos de la clase trabajadora de una parte del planeta (Norte), fue el producto de un proceso imbricado por los factores de modernidad, (neo)colonialismo y desarrollo. De este modo, se presenta la urgencia de deconstruir el sistema económico actual ecologicida y genocida, que ha sido el creador de este nuevo orden mundial que ha rentabilizado la sobreexplotación y la muerte de miles de mujeres. Así, esta investigación bebe de la aplicación de un enfoque teórico-metodológico interseccional, es decir, es necesario entender la subordinación de las mujeres a partir del conjunto de variables co-constitutivas (género, raza, sexualidad, espiritualidad, etcétera) y desde los conocimientos situados de Donna Haraway. Respecto a la estructura, en la primera parte del artículo se presentará una crítica al discurso mainstream de las Relaciones Internacionales desde la perspectiva descolonial. Posteriormente, se estudiará la relegación del trabajo reproductivo a las mujeres vinculada al proceso colonial, además, se pretenderá demostrar los efectos del sistema económico internacional en las vidas de las subalternizadas, sobre las mujeres trabajadoras, las racializadas, las colonizadas, las refugiadas, las trans o las migrantes. En último término, se presenta un diálogo entre los feminismos descoloniales y la economía feminista para repensar y justificar el bienestar como camino hacia la protección de la vida planetaria. En definitiva, el contexto global es un sistema que le ha cedido la batuta a un modelo que imposibilita garantizar el cuidado de las vidas como consecuencia de su naturaleza eurocéntrica, racista, colonial, heteropatriarcal, ecologicida y un largo etcétera. Por esta razón, este artículo abre una puerta al diálogo entre los feminismos descoloniales y la economía feminista para tratar de encontrar consensos que permitan crear una agenda feminista, subversiva y común. Para este camino de reflexión y cuestionamiento la presencia de las Relaciones Internacionales se vuelve indispensable. Esta disciplina debe acompañar, desde el inicio, la fase de transición que consiga desplazar el capital para situar en el epicentro del sistema los cuidados y la sostenibilidad de la vida.
\end{abstract}

\section{Palabras clave}

Relaciones Internacionales; feminismos descoloniales; interseccionalidad; cuidados; sostenibilidad de la vida.

\section{TITLE}

International Relations from the decolonial feminisms. A dialogic approach to a decolonial feminist economy

\begin{abstract}
Since the mid-twentieth century, the modern-colonial capitalist system has been consolidated by a Eurocentric logic that has aggravated the North-South gap. Thus the international economic relations that imposed the generation and racialization of labour were forged. In this sense, the assurance of the socio-economic rights of the working class of the Global North was the product of a process imbricated by the factors of modernity, (neo)colonialism and development. Therefore, the urgency of deconstructing the current ecocide and genocidal economic system is presented, for this new world
\end{abstract}

\section{DOI:}

http://doi.org// 0.15366/relacionesinternacionales2020.44.008

Formato de citación recomendado:

PIZARRO GÓMEZ, Selena (2020), “Las Relaciones Internacionales desde los feminismos descoloniales. Una propuesta dialógica hacia una economía feminista descolonial”, Relaciones Internacionales, n 44, pp. I47-I64.

\section{* Selena} PIZARRO GÓMEZ, Periodista por la Universidade de Santiago de Compostela (USC) y Máster en Derecho Internacional y Relaciones Internacionales.

Recibido: 30/01/2020 Aceptado: 06/04/2020 
order has profited from the overexploitation and death of thousands of women. This investigation implements a theoretical-methodological intersectional approach, that is to say, to understand the subordination of women there is a need to do so from a set of co-constitutional variables (gender, race, sexuality, spirituality, etc.) and from "situated knowledges" as Donna Haraway puts it. This perspective allows us to go beyond gender oppression, for which it will be essential to actively listen to the experiences of other women who have been marginalized and excluded by hegemonic and Eurocentric feminisms, only considered as objects of study never as political subjects. This work is implicated in the will to study and move towards an alternative reading of international relations. For this purpose, it is my proposal to begin in the feminist margins of decolonial feminisms, from the ideas of thinkers who are characterized by not seeking a consensus but a conversation from difference. Regarding the structure, the first part of the article will present a critique of mainstream international relations discourse from a decolonial perspective. Thus, the aim is to prove through a critique of the hegemonic paradigm that international relations serves the interests of the Global North as a consequence of Eurocentric thinking. Subsequently, the relegation of reproductive work to women linked to the colonial process will be studied. Furthermore, it will seek to demonstrate the effects of the international economic system on the subalternized, racialized, and colonized lives of workers, refugees, or migrants. In relation to this issue, the study and review of historical factors is fundamental because international relations cannot be understood without studying history; that is, the creation of the current international economic system as a consequence of the construction of the international and sexual division of labour and the processes of colonization and racialization. In turn, the above study has as an objective to demonstrate that the care economy is the backbone of the functioning of the international economic system. In other words, if women - traditionally responsible for maintaining lives - went on general strike, the world economy would come to a standstill. Likewise, the violence caused by the modern/colonial capitalist system on the bodies of the subalternized will be analyzed. In this sense, the epistemologies of the South become essential for the study of the neocolonial North-South economic relations where violence against women plays a key role. Examples of this are free-trade zones, extractivism, or in the worst of the cases: wars. Finally, a dialogue between decolonial feminisms and the feminist economy is presented to rethink and justify welfare as a path towards the protection of planetary life. In short, the global context is a system that has ceded the baton to a model that makes it impossible to guarantee the care of lives as a consequence of a nature that is Eurocentric, racist, colonial, heteropatriarchal, ecocidal and so forth. The proposal to urge an alternative is justified through a crisis of a systemic nature which, despite attempts to blur its permanence, is still present through political and socio-economic conflicts. Thus, the Global North is suffering from a process leading to areas that were once part of the centre are now peripheral - as a consequence of the globalised crisis and increased by austerity policies. This consolidates a political, economic, ecological and ethical crisis, which forces us to question the direction in which we are navigating and how we will manage this process, even if this seems inevitable with respect to environmental degradation and being immersed in a context of social hyper-segmentation, where growing inequalities seem to be naturalized and at the same time legitimized. For this reason, this article aims to establish a dialogue between descolonial feminisms and feminist economics to seek a consensus for the creation of a feminist, subversive and common agenda. For this sort of reflection and questioning the presence of international relations becomes indispensable. From the beginning, this discipline should go hand in hand with the transition phase aimed at replacing capital with the care economy and sustainability of life as the epicentre of the system. This research seeks to outline the nonconformity of accepting that history has already been written against those who prevent us from dreaming of the change we want and believe in. But why now? The present moment is decisive. In the face of the threat to planetary life from a destructive economic system, it is more necessary than ever to participate in the creation of another paradigm of international relations through other knowledges. Undoubtedly, the image of the Amazon in flames is further proof of the urgency of initiating a transformation of the global political and socio-economic system. From where and for what purpose is knowledge produced? What role does the economy play within international relations? Who benefits and who is harmed by the globalized capitalist model? Where do women stand within the economic system? Which lives are worth living? Is it possible to initiate an alternative to capitalism from Europe? These questions are not posed with the aim of giving a definitive answer, but with the intention of provoking dialogue and reflection. That is to say, against the logic of the ethics of war, it is manifested to promote the transition of the current international economic system towards a new model for which it will be essential to initiate an analysis of international relations from feminist genealogies and from decolonial thought.

\section{KEYWORDS}

International Relations; decolonial feminisms; intersectionality; care; sustainability of life. 


\section{ntroducción}

Uma sobe e puxa a outra, dice uno de los lemas de la Marcha das Mulheres Pretas do Brasil.Así empecé esta investigación, empujada por la fuerza de los movimientos feministas que ya en el siglo XXI se consolidan como transnacionales y heterogéneos. Por esta razón política y ética, presento la necesidad de promover una lectura diferente de las Relaciones Internacionales, un espacio donde históricamente se ha marginado, o directamente excluido, a las mujeres. Mientras, los feminismos han logrado mostrar cómo el discurso mainstream de esta disciplina es exiguo para comprender la complejidad de la realidad.

El pensamiento hegemónico de las Relaciones Internacionales ha focalizado el estudio en la seguridad y la cooperación económica desde el Norte Global', consolidadas como las dos grandes preocupaciones en la agenda de investigación. Intencionadamente se han ocultado cuestiones fundamentales para el análisis de las Relaciones Internacionales, pero que las teorías feministas han exigido y han servido para cuestionar las visiones ahistóricas de la creación del sistema internacional y la aparición del Estado-nación.

Por consiguiente, el objetivo de este artículo es intentar establecer un diálogo entre los feminismos descoloniales y la economía feminista — profundamente occidental ${ }^{2}$ - , para crear consensos que contribuyan a la defensa de la vida en su conjunto. En este sentido, el trabajo reproductivo y de cuidados, que son "las actividades y relaciones gracias a las cuales nuestra vida y nuestra capacidad laboral se reconstruyen a diario" (Federici, 20I3, p. 2I), constituye un elemento clave para la sostenibilidad de la vida. Por ello, será necesaria una revisión histórica sobre cómo este trabajo no remunerado ha sido privatizado y relegado a las mujeres a través de los feminicidios, así como, la sobreexplotación y domesticación de sus cuerpos.

De este modo, el trabajo reproductivo ha sido una actividad fundamental en la configuración del sistema capitalista moderno/colonial ${ }^{3}$; un sistema que destruye la vida al sostenerse sobre procesos ecologicidas ${ }^{4}$ y que, además, ha sido el inventor de ese "Tercer Mundo" 5 .Así, se presenta

\footnotetext{
Los conceptos de Norte y Sur sirven para sustituir a otros como Primer Mundo y Tercer Mundo o países desarrollados y países en vías de desarrollo o subdesarrollados. Según Boaventura de Sousa el Norte es entendido como la fuente de colonialidad, sufrimiento, violencia, humillación e injusticia, y el Sur como los receptores de estas manifestaciones, implica "una metáfora del sufrimiento humano sistemáticamente causado por el colonialismo y el capitalismo” (Medina Martín, 20I2, p. 2I7).

2 Es necesario tener presente que la economía feminista se consolidó en el contexto occidental a partir de la década de los setenta —a pesar de que las primeras voces disidentes aparecieron a lo largo del siglo XIX-; no obstante, no fue hasta los años noventa con la celebración de la Conferencia Anual de la American Economic Association (1990) y la creación de la International Association For Feminist Economics (IAFFE) en 1992 cuando asentó sus bases (Carrasco, 2006, p. 29).

3 En esta investigación me referiré al sistema capitalista moderno/colonial como síntesis de un concepto más amplio que recoge las jerarquías globales dominantes, definido por Grosfoguel como "sistema-mundo capitalista/patriarcal occidentalocéntrico/cristianocéntrico moderno/ colonial" (2002, pp. 203-224). Así mismo, utilizaré la categoría de sistema capitalista moderno/colonial en oposición a la noción de Estadonación, al entender que esta última es la unidad de análisis de las ciencias sociales eurocéntricas y que "reduce la matriz temporal/espacial del análisis al tiempo histórico de las fronteras jurídico-políticas de los Estados-naciones creados hace apenas unos doscientos años, y en algunos casos hasta menos de cien años" (Grosfoguel, 20I6a, p. I55).

4 En este artículo utilizaré el concepto ecologicidio o ecologicida refiriéndome a los procesos de destrucción planetaria provocados por el sistema capitalista moderno/colonial, en este sentido, "las consecuencias pasan no solamente por la destrucción ecológica, sino también por la violencia que usan para desplazar a seres humanos de sus territorios” (Grosfoguel, 20I6b, p. I27). Así mismo, el Movimiento de Mujeres Indígenas por el Buen Vivir utiliza el concepto "terricida”. Según Millán, weychafe mapuche: “El terricidio (...) refleja la visión tridimensional de la vida de los pueblos indígenas. Lo que se conoce como el medio ambiente, nosotros lo llamamos ecosistemas tangibles que están siendo devastados, contaminados. Pero también la aniquilación de los pueblos, el asesinato selectivo contra líderes indígenas, con la eliminación de los pueblos originarios que al ser asesinados, perseguidos, criminalizados y encarcelados. Implica también la eliminación de un modo de entender el arte de habitar, de poder contribuir a la creación de una nueva matriz civilizatoria" (Mayorquín, 2020).

5 Véase la obra de Escobar (2007).
} 
también la necesidad de incorporar un análisis crítico desde el pensamiento descolonial en la academia de las Relaciones Internacionales. Ya que, cuestionar el sistema implica también desvelar el discurso dominante que refleja el vínculo foucaultiano entre saber y poder.

Por esta razón, será imprescindible partir de la genealogía feminista, no sólo para el respeto y bienestar de las vidas humanas, sino para el futuro del conjunto planetario. Así, la crítica a ese sistema de rapiña exige de manera ineludible la incorporación de los feminismos descoloniales, que beben de múltiples corrientes dando lugar a un conjunto de epistemologías otras ${ }^{6}$. Estos pensamientos surgen desde los márgenes como una nueva apuesta en el estudio de las Relaciones Internacionales. Para Espinosa Miñoso, los feminismos descoloniales reinterpretan "la historia en clave crítica a la modernidad, ya no sólo por su androcentrismo y misoginia, como lo ha hecho la epistemología feminista clásica, sino dado su carácter intrínsecamente racista y eurocéntrico" (2012, p. 144).

Además, los feminismos descoloniales consiguen articular la perspectiva de la "interseccionalidad" —acuñada por Kimberlé Crenshaw en 1989 y desarrollada desde los feminismos negros - que se basa en una propuesta metodológica y teórica, "una apuesta por generar prácticas que evidencien los privilegios sin caer en la victimización” (Ruiz Trejo y Betemps, 20I4, p. 174). Es decir, la interseccionalidad se refiere al análisis de las experiencias de opresión de las mujeres a partir del conjunto de variables coconstitutivas (género, raza, sexualidad, espiritualidad, etcétera), situado y ligado a un contexto específico. Este enfoque será imprescindible para un análisis de las vidas subalternizadas ${ }^{7}$, es decir, las vidas de las mujeres trabajadoras, empobrecidas, racializadas, migrantes, refugiadas, colonizadas, lesbianas, trans, etcétera.

Por otro lado, la incorporación de la economía feminista en esta investigación se debe a su contribución histórica y política en el cuestionamiento del sistema económico internacional. En este sentido, la expansión del capitalismo y el proceso de colonización están relacionados con la imposición global del trabajo reproductivo y de cuidados a las mujeres, se trata por tanto de repensar el sistema económico internacional desde la mirada feminista.

Esta investigación pretende poner de manifiesto que entender la economía desde los feminismos descoloniales implica también analizar las relaciones de poder entre géneros, razas o clases, como elementos constitutivos del sistema socioeconómico. Este artículo busca recordar que las alianzas contra el sistema capitalista moderno/colonial serán fundamentales para la construcción de esa nueva agenda basada en un proyecto feminista común y transnacional, en el que el interés general sea el posicionamiento de la vida en el centro del sistema.

La importancia de esta propuesta dialógica desde el Norte - como un humilde aporte teórico - reside también en la responsabilidad que tenemos de participar en el desvelamiento de este sistema de muerte tras imponerse desde nuestro propio territorio, desde Europa, al resto

\footnotetext{
6 La expresión "pensamientos y epistemologías otras" hace referencia al "paradigma otro" que analiza Mignolo (2003) en su obra.

En esta investigación me referiré a las "vidas subalternizadas" exclusivamente en relación a las mujeres respecto a las distintas matrices de opresión que van más allá de la discriminación sexo-género. No obstante, es necesario entender el lado subalterno como aquel en el que se ubican las vidas del Sur Global, de las mujeres, de la clase obrera, de los cuerpos racializados/colonizados, los no capacitistas, los que se pronuncian desde las disidencias sexuales y de género. Es decir, las vidas subalternizadas son aquellas que no forman parte del patrón hombre, blanco, burgués, urbano, heterosexual y del Norte Global.
} 
de pueblos y civilizaciones. Así mismo, asumo la problemática y la dificultad de no reducir a las otras mujeres a una categoría monolítica, tal y como ya criticó Mohanty en 1984 a las feministas occidentales. El objetivo es investigar "con", para dejar de investigar "sobre" (Gimeno, citado en Medina Martín, 2019, p. I19). Solo a partir de ese reconocimiento podremos iniciar un proceso de deconstrucción y descolonización de las ciencias sociales, y en concreto de las Relaciones Internacionales.

\section{Crítica al discurso eurocéntrico de las Relaciones Internacionales}

El nacimiento de las Relaciones Internacionales está estrechamente vinculado con la historia diplomática y el derecho internacional, a partir del Tratado de Westphalia en 1648 con la creación del Estado moderno o Estado-nación en Europa, que implicó un cambio en el orden feudal y la aparición del sistema internacional. Sin embargo, el estudio de la disciplina no llegó hasta el fin de la Gran Guerra impulsado por el progreso de las telecomunicaciones, la importancia de la resolución pacífica de los conflictos bélicos y la Revolución Industrial que surgió desde Inglaterra.

El pensamiento dominante de las Relaciones Internacionales afianzó el discurso eurocéntrico ${ }^{8}$; como consecuencia de la hegemonía estadounidense en la política internacional y en la producción de conocimiento científico, factores que se consolidaron tras la caída del Muro de Berlín en 1989 (Barbé y Soriano, 20 I5, p. 142). Las tensiones políticas entre Estados Unidos y la Unión Soviética impulsaron a la academia estadounidense a desarrollar una teoría que consiguiese dar respuestas a sus problemas para mantener la hegemonía dentro del sistema bipolar.

El análisis del pensamiento dominante de las Relaciones Internacionales, que sirve a los intereses de las potencias mundiales y consolida la brecha Norte-Sur, revela el vínculo foucaultiano entre saber y poder. De este modo, las teorías se construyen a partir de un contexto social y político concreto y localizado. Es decir, la dialéctica saber/poder se refleja en el mainstream de las Relaciones Internacionales, que desde los nichos de la academia ha excluido el género y la raza para explicar la realidad del mundo. Según Moreno Rodríguez, "este hecho devela la capacidad de la disciplina de jugar un papel de permisor epistémico a la hora de reconocer o silenciar las voces otras" (2019, p. 236). No obstante, en las últimas décadas han aparecido distintas corrientes de pensamiento crítico que han cuestionado el discurso mainstream de las Relaciones Internacionales.

Por esta razón, tras identificar el paradigma hegemónico en las Relaciones Internacionales surge la necesidad de construir un discurso otro. La crítica principal es al pensamiento eurocéntrico en el que se fundamenta la narrativa dominante y su contribución a la creación de las desigualdades latentes en la división de los hemisferios Norte y Sur, pero también a través del sistema de jerarquización de género, raza y clase. En este sentido, se trata de cuestionar el discurso mainstream y razonar sobre cómo los Estados-nación ya no son los principales actores

\footnotetext{
"A pesar de que Barbé y Soriano (20I5) y otras/os autoras/es de las Relaciones Internacionales emplean el concepto "americanocentrismo" para analizar el discurso mainstream de la disciplina, en este artículo se utiliza "eurocentrismo" en coherencia con la perspectiva feminista descolonial. La adopción de este término es una crítica al análisis dominante de las teorías de las Relaciones Internacionales, que se ha centrado en el estudio de la disciplina a partir de 1945 desde Occidente, ignorando de este modo los efectos del colonialismo y la construcción de la modernidad. No obstante, creo necesario aclarar mi propia incomodidad con la expresión "americancéntrico" para referirse al papel dominante de Estados Unidos, pues afianza la usurpación que se viene haciendo en este país y que en otras lenguas se acepta —por ejemplo el francés, que usa la expresión americain para referirse a la población de Estados Unidos-. Por ello, rechazo el uso del nombre de todo un continente para referirse a un solo país y dar a la expresión América la impronta de sus políticas, intereses, etcétera; superando incluso la dimensión colonialista que pueda tener el nombre que dio Europa a ese territorio, ignorando los nombres que ya tenía, como Abya Yala.
} 
internacionales; no solo por la aparición de nuevos actores no estatales, sino por la complejidad de las relaciones que se establecen como consecuencia del sistema capitalista moderno/colonial, y que provoca que el marco de referencia tenga que ampliarse.

El eurocentrismo se implantó cuando Europa se colocó a sí misma en el centro del sistema y al resto de sociedades en las periferias. Es decir, se centralizó la experiencia históricosocial europea y se expandió la idea del fundamentalismo eurocéntrico como "la premisa de que hay sólo una tradición epistémica desde la que se pueden alcanzar la Verdad y la Universalidad" (Grosfoguel, 2006, p. 20). Así mismo, el eurocentrismo escapa de su referencia geográfica, ya que de forma más compleja explica la construcción del pensamiento hegemónico vinculado al imperialismo y al capitalismo globalizado. En otras palabras, se refiere a "un proyecto de dominación política, epistémica y económica" que configura la realidad social, es decir, la realidad se construye discursivamente (Garcés, 2007, p. 222). Por lo tanto, cabe poner de relieve la importancia de "la ubicación, no sólo geo-histórica sino también epistémica (y el correlato entre ambos), del pensamiento" que construye el imaginario político y social (Mignolo 2007, p. 42).

Con todo, la perspectiva eurocéntrica del discurso hegemónico concibe el mundo actual como descolonizado y poscolonial ${ }^{9}$. Aunque en el siglo $\mathrm{XIX}$ las colonias españolas iniciaron el proceso de descolonización centrado en la independencia jurídico-política de los territorios periféricos ocupados y que, más tarde, imitaron las colonias inglesas y francesas, este proceso fue incompleto. De manera que, actualmente, nos encontramos en una situación global de neocolonialidad contemporánea que está basada en "la dominación y explotación económica del Norte sobre el Sur" (Castro-Gómez y Grosfoguel, 2007, p. 17). En este sentido, la modernidad representa un proyecto emancipatorio y civilizatorio que se sostiene sobre la explotación y el expolio de la zona del no $\operatorname{ser}^{10}$, y que se perpetúa a través de la "colonialidad del conocimiento". Para Mignolo, "el dominio entero del conocimiento ha sido apropiado y manejado por las instituciones europeas y estadounidenses operando en lenguajes imperiales" (2005, p. 55).

Así mismo, los feminismos descoloniales desvelan el discurso de colonialidad de las Relaciones Internacionales y cómo la misoginia se convirtió en un elemento fundamental para la modernidad". De este modo, Lugones (2008) analiza el "sistema moderno-colonial de género" que consiste en una organización social basada en el dimorfismo biológico - la naturalización del sexo como una condición binaria-, el régimen del heterosexualismo y el patriarcado ${ }^{2}$. En otras

\footnotetext{
Existe una tendencia de pensamiento universal que concibe el mundo actual como descolonizado y poscolonial respecto al ámbito jurídicopolítico, fundamentalmente a partir de la creación de las Naciones Unidas. Sin embargo, en esta investigación es necesario aclarar el error de esta afirmación atendiendo a los ejemplos del Sáhara Occidental y Palestina, pues ambos territorios se encuentran actualmente bajo ocupación.

10 Grosfoguel (2002) analiza la línea de lo humano o la zona del ser, como aquella donde se hallan las personas humanas, hiperhumanizadas; $y$ bajo la línea inferior aparece la zona del no ser, deshumanizada y habitada por tanto por subhumanas y no-humanas. En este sentido, cabe poner de relieve cómo la opresión de género constituye un privilegio de la mujer blanca, pues las mujeres que habitan en la zona del no ser no son consideradas mujeres, sino hembras. Esta perspectiva del género/sexo dentro de la teoría del sistema mundo es introducida por Lugones (2008).

"En esta línea, Ochoa Muñoz considera que el sistema capitalista moderno/colonial se erigió sobre tres formas de violencias interrelacionadas: "la esclavitud (bestialización), la racialización (de las poblaciones colonizadas) y la feminización de los indios (que incorpora el sexismo y la misoginia)"; el proceso de deshumanización de las personas indígenas sirvió para negarlas como sujetas, por medio de "la feminización y el uso de una violencia misógina-genocida contra las poblaciones colonizadas y conquistadas" (20I4).

12 "Rita Segato sistematiza la noción de colonialidad del género, introduciendo una importante crítica al planteamiento de Lugones. Cuando Lugones propone que las relaciones de poder y las categorías de género tal como las conocemos hoy, son una invención de la colonial/ modernidad, estaría presuponiendo un pasado precolonial en el que las sociedades tenían mayor grado de igualdad en las relaciones entre
} 
palabras, "la fusión de la idea de raza y la idea de género" permitieron la expansión del capitalismo y la imposición del modelo de democracia liberal en los países occidentales, fenómenos sobre los que se fundamenta el pensamiento eurocéntrico, por ello, "debemos hablar no solo de la colonialidad de género sino incluso de la colonialidad de la democracia liberal" (Mendoza, 20I4, p. 96).

La noción de "colonialidad de la democracia liberal" se refiere al proceso de racialización y engenerización que sufrió el trabajo, y que permitió la creación de la democracia liberal en Occidente a partir de ese gran pacto social entre "capitalistas y la clase obrera masculina de origen europeo (blancos) en detrimento de los trabajadores no asalariados no blancos" (Mendoza, 20I4, p. 96). Las feministas descoloniales plantearon cómo el trabajo engenerizado y racializado, en parte, permitió el bienestar de las democracias liberales occidentales, a partir del pacto entre el sistema capitalista, la clase obrera y los feminismos hegemónicos.

De este modo, los feminismos descoloniales se enfrentan a la "colonialidad global"13 cuestionando las epistemologías dominantes que han jugado un rol principal en la construcción de la modernidad, a través de prácticas epistemicidas que han universalizado el saber occidental y han barbarizado los conocimientos otros. Es decir, la subalternización de las epistemologías del Sur se produjo gracias a la colonización territorial, política y económica, así como, a través de la "violencia epistémica" (Spivak, 20I0).

Occidente por medio de la expansión del capitalismo y el colonialismo decidió cuál sería la interpretación de la realidad, consolidó las únicas formas de ser, de pensar y de saber.Por esta razón, surge la necesidad de iniciar, a través de la genealogía feminista, un proceso de descolonización y despatriarcalización de las Relaciones Internacionales, porque la narrativa histórico-política dominante sostiene el sistema capitalista moderno/colonial.

\section{Análisis feminista de las vidas subalternizadas}

El trabajo reproductivo y de cuidados constituye uno de los factores principales en el desarrollo del sistema capitalista moderno/colonial. Por esta razón, se parte del estudio del proceso de confinamiento de las mujeres en los hogares a partir de uno de los cuatro genocidios/epistemicidios ${ }^{14}$ desarrollados a lo largo del siglo XVI — y que sirvieron para que el sistema capitalista moderno/ colonial alcanzara su internacionalización-, se trata del asesinato masivo de mujeres en Europa quemadas vivas bajo acusaciones de brujería.

Las economías occidentales fueron forjadas sobre el genocidio, la esclavitud y el racismo a

\footnotetext{
los géneros. Ante esta perspectiva, Segato plantea que entre las comunidades americanas y africanas previas a la 'intrusión' colonial, existía una organización patriarcal de baja intensidad, diferente a la del género occidental, que sin embargo es capturada, intensificada y reorganizada desde dentro con la llegada de la modernidad-colonial” (Bidaseca, 2019, p. 21 ).

13 El concepto de "colonialidad global" hace referencia al análisis de Quijano respecto a "la parte invisible y constitutiva de la modernidad vinculando expresamente la colonialidad del poder en las esferas políticas y económicas con la colonialidad del conocimiento como instrumento imperial de colonización", además, la fase de colonialidad surge a partir de 1945 y se mantiene a pesar de los procesos de descolonización jurídico-política (Medina Martín, 2016, p. 85).

14 Desde el pensamiento del giro decolonial se distinguen cuatro genocidios/epistemicidios durante el siglo XVI, contra "los musulmanes y los judíos en la conquista de Al-Andalus en nombre de la 'pureza de sangre'; contra los pueblos indígenas primero en el continente americano y luego los aborígenes en Asia; contra los africanos con el comercio de cautivos y su esclavización en el continente americano; contra las mujeres que practicaban y transmitían el conocimiento indo-europeo en Europa, quienes fueron quemadas vivas acusadas de brujas" (Grosfoguel, 2013, p. 39).
} 
partir del siglo XV. La memoria de la caza de brujas de Federici (20l0) revela cómo el proceso de expansión del capitalismo y del colonialismo se sostuvo a partir de una guerra contra las mujeres al principio de la era moderna. Los feminicidios sirvieron para la domesticación de los cuerpos y el control de la reproducción, así, las mujeres fueron confinadas a la esfera privada, relegadas al trabajo invisibilizado dentro de los hogares.

En el siglo XV las mujeres se convirtieron en el principal objetivo, en la mayor manifestación de lo hereje. Incluso, se llegó a justificar la violación a aquellas que formaban parte de la clase obrera, hasta convertirse en una práctica consentida por el estado sin apenas castigo o condena en distintas ciudades de Europa occidental. La legalización de la violación provocó el debilitamiento de la lucha antifeudal e "insensibilizó a la población frente a la violencia contra las mujeres, preparando el terreno para la caza de brujas que comenzaría en ese mismo periodo" (Federici, 2010, p. 79).

Durante el feudalismo se produjo un proceso constante de luchas de clase y una vez finalizado se llevó a cabo la gran división social. El asesinato masivo de mujeres quemadas en la hoguera fue el resultado de la lucha antifeudal del movimiento campesino en Europa, y sus muertes cuentan cómo este sistema ha sido construido. Los feminicidios fueron la consecuencia del peligro que implicaba la resistencia de las mujeres para el desarrollo del capitalismo. Por ello, sus cuerpos fueron explotados y violados para servir como herramienta de reproducción y de acumulación.

A su vez, es imprescindible vincular el destino de las mujeres en el continente europeo con el de las personas racializadas y colonizadas en el territorio americano ${ }^{15}$. De este modo, se refleja una profunda relación entre la división sexual de roles y la emergencia eld capitalismo/ colonialismo, que contribuyó a la feminización de la pobreza a nivel internacional.Ya que el proceso de globalización económica implicó la relegación de las mujeres a los trabajos más precarizados y al confinamiento en el espacio doméstico.

Mientras que en Europa, tras la cacería de brujas, la domesticación se fundamentó en "separar a las mujeres de la esfera productiva al mismo tiempo que las convirtió en amas de casa u obreras explotadas"; en los territorios colonizados consistía en "las violaciones masivas a mujeres indígenas como instrumento de guerra de conquista y asentamiento colonial, la pérdida de su estatus social y político, esclavización, reducción a servidumbre y la intensidad letal del trabajo" (Mendoza, 20I4, p. 95).

De acuerdo con Mendoza, la domesticación de las mujeres blancas y no blancas ocupó un papel clave en la división del trabajo asalariado, así, a la marca de "la jerarquía racial" añade la marca de "la jerarquía de género" del trabajo asalariado (Bidaseca et. al., 20I5, p. 209). Además, existe una clara relación, entre el confinamiento de las mujeres en los hogares y la violencia, que se visibiliza en los feminicidios ejecutados por el proyecto civilizatorio de la modernidad.

"Los feminicidios, como prácticas casi maquinales de extermino de las mujeres son también una invención moderna. Es la barbarie de la colonial modernidad

\footnotetext{
15 Federici compara en su obra el destino de las mujeres europeas acusadas de brujería con el de las personas esclavizadas — tanto africanas como indígenas americanas-, que fueron sometidas en la construcción de las plantaciones del territorio colonizado y explotadas para el desarrollo del sistema capitalista, con especial impacto sobre las mujeres (2010, p. 272).
} 
(...) Su impunidad, como he intentado argumentar en otro lugar, se encuentra vinculada a la privatización del espacio doméstico, como espacio residual, no incluido en la esfera de las cuestiones mayores, consideradas de interés público general. Con la emergencia de la grilla universal moderna, de la que emanan el Estado, la política, los derechos y la ciencia, tanto la esfera doméstica como la mujer que la habita, se transforman en meros restos, en el margen de los asuntos considerados de relevancia universal y perspectiva neutra" (Segato, 2016, p. II7).

En definitiva, los úteros de las mujeres fueron utilizados por el sistema capitalista moderno/ colonial para crear la fuerza de trabajo, y mediante el control y la domesticación consiguieron naturalizar y sexualizar los cuidados. Esta relegación del trabajo reproductivo a las mujeres y a la esfera privada fue una estrategia que permitió también la ocultación del "conflicto capital-vida"16 y se convirtió en la realidad contemporánea. De este modo se niega a las mujeres cuidadoras el reconocimiento de sujetas políticas, lo que impide que se gestione el conflicto, traspase los hogares y se politice. No obstante, el contexto del confinamiento de las mujeres en los hogares, durante el desarrollo de las sociedades industriales, se escapa de las experiencias de las mujeres que también trabajaban fuera del ámbito de los hogares, principalmente, de las empobrecidas, las racializadas o las colonizadas.

\section{I. La invisibilización y feminización de los cuidados en el Norte}

El trabajo reproductivo y de cuidados se consolida como la llave para la sostenibilidad de la vida, a pesar de su privatización y relegación al ámbito de los hogares, no obstante, se debe entender esta crítica en un contexto del Norte Global urbano. En primer lugar, es necesario aclarar la idea de opresión del hogar y la familia como un rasgo distintivo en la diversidad de experiencias de las mujeres. En este sentido, los feminismos descoloniales cuestionan esta visión como una realidad universal desde los feminismos hegemónicos, puesto que "para muchas mujeres negras quienes sufrieron durante generaciones la esclavitud y luego la marca indeleble de la mirada racializada, la posibilidad de tener una familia estaba negada, así que constituirla era toda una conquista" (Quiroga Díaz, 20I2, p. II2) 17 .

Es decir, mientras que para muchas mujeres la familia era defendida como un derecho, las feministas del Norte la denunciaban como una forma de opresión producto de la revolución francesa. Esto también justifica la importancia de la interseccionalidad en el análisis y la necesidad de su incorporación en la economía feminista, que hasta ahora ha asumido varias categorías de opresión eurocéntricas como, por ejemplo, la universalización del relato de la familia como opresión de todas las mujeres.

Ahora bien, desde el Norte Global urbano, el trabajo asalariado para las mujeres pasa a ser una extensión de su ya naturalizado trabajo reproductivo. Así, a partir de la glorificación de la familia se consigue recluir este trabajo al ámbito privado; es decir, la organización de la familia nuclear es uno de los asuntos más importantes para la agenda capitalista a partir de la división del trabajo (Federici, 2013, p. 62).

\footnotetext{
16 Para Pérez Orozco el concepto "conflicto capital-vida" sirve para explicar que "el actual sistema socioeconómico hoy se erige sobre una tensión estructural entre la acumulación de capital y la sostenibilidad de la vida que es irresoluble, aunque se intente acallar" (20I4, p. I02).

17 Sobre la articulación raza/trabajo véase el trabajo de Quijano (2014).
} 
La cuestión fundamental reside en la necesidad del sistema capitalista moderno/colonial de mantener la invisibilización de determinadas esferas económicas, como ocurre con el trabajo reproductivo con el fin de incorporar dentro el conflicto capital-vida. De esta manera, dicha problemática no aparece como un asunto a resolver en la esfera económica al ser relegada a sujetas no políticas, fuera del ámbito público.

Con todo, existe una conexión directa entre los cuidados y las desequidades, en un doble sentido: (a) la degradación social de los cuidados provoca que esta actividad sea atribuida a quienes se encuentran en una situación de mayor vulnerabilidad; y (b) la calidad de los cuidados dependerá de la situación socioeconómica, que dependerá, a su vez, de la jerarquización de las diferencias. Así, se configura un vínculo entre los rasgos característicos de los sistemas de cuidados y los del sistema capitalista, que conducen a la privatización de la sostenibilidad de la vida.

De este modo, se evita la colectivización de la responsabilidad del trabajo reproductivo y sirve para promover su feminización, invisibilización y despolitización. En otras palabras, el sistema capitalista moderno/colonial se ha beneficiado de la imposición del binarismo de género - que a su vez es racializado y relacionado con la clase, junto a otras variables coconstitutivas-, para la asociación de los hombres "a una ética productivista" y de las mujeres a "una ética reaccionaria del cuidado" (Pérez Orozco, 20 I4, p. 168).

En síntesis, el posicionamiento hegemónico de los mercados en el sistema económico internacional generó el conflicto capital-vida. De hecho, la respuesta neoliberal surgida de la alianza entre los mercados y los estados, que se dio al estallido de la crisis económica de 2007, consistió en el ataque a la vida a través de la gran estafa de la deuda, cuyo origen se encuentra en los programas de ajuste impuestos en los territorios de América Latina durante los años ochenta (Pérez Orozco, 20l4, p. 96). Es decir, el conflicto capital-vida se consolida a través de tres lógicas encargadas de mantener las desequidades sociales: la acumulación de capital, los cuidados y la preservación de una vida digna.

\subsection{Las vidas subalternizadas más allá del Norte}

Durante el siglo $\mathrm{XX}$, las mujeres se han convertido en las protagonistas de las migraciones del Sur hacia el Norte, sobre todo para formar parte del sector servicios y el trabajo del hogar. En los países occidentales, tras la incorporación en masa de las mujeres al trabajo asalariado, gracias a la introducción de las tecnologías, el trabajo reproductivo en el lugar de sufrir un proceso de tecnificación ha sido "redistribuido y cargado sobre las espaldas de diferentes sujetos mediante su comercialización y globalización” (Federici, 2013, p. 175).

Por su parte, Quiroga Díaz considera que la integración de la mano de obra femenina a los países del Norte generó "una reasignación en términos de clase (...) que alentó la migración de mujeres pobres de la periferia, en primera instancia, para ocuparse de actividades reproductivas y domésticas" (2009, p. 80). Se consolida así un proceso de feminización de la migración de las poblaciones del Sur, como consecuencia de la crisis de reproducción y la demanda de los países del Norte. Además, los puestos laborales que pasan a ocupar estas mujeres son aquellos caracterizados por la precariedad salarial y por estar relacionados con actividades rechazadas socialmente. 
La migración se convierte en un factor esencial para el proceso capitalista a partir de la acumulación de las clases trabajadoras; siendo las mujeres africanas, asiáticas y latinoamericanas una parte fundamental de este movimiento migratorio que se convierte en mano de obra. Los gobiernos del Norte han conseguido ahorrar sumas importantes de dinero a través del desplazamiento del trabajo reproductivo a las mujeres del Sur —principalmente—; sin cuestionar las graves consecuencias que esto genera sobre las vidas de las migrantes y sus comunidades de origen. En palabras de Hochschild, se produce la "transferencia global del cuidado y las emociones" (citado en Federici, 20I3, p. 2 I0) que construye las cadenas globales de cuidados.Así, Occidente ha propiciado "una solución colonial a la cuestión del trabajo reproductivo" (Federici, 20I3, p. I22).

En este sentido, es importante entender la creación de las cadenas globales de cuidados a partir del proceso de interrelación entre la crisis de cuidados en el Norte con la crisis de reproducción social en el Sur. Es decir, en el Norte se produce una crisis de cuidados consecuencia de la sobrecarga que se da en los hogares y que termina por consolidar la sobreexplotación de las mujeres. Mientras que, al mismo tiempo, se agrava una crisis de reproducción social en el Sur que se traduce en la feminización de las migraciones y la creación de las cadenas globales de cuidados; es decir, se crea un proceso de internacionalización del trabajo reproductivo.

\section{Una mirada descolonial de las relaciones económicas Norte-Sur}

El sistema capitalista moderno/colonial se consolidó a partir de la creación de un mercado global que ha perseguido el dominio del trabajo y la expropiación de las riquezas, por medio de la explotación y la colonización. Por consiguiente, las relaciones económicas subordinan al resto de relaciones y configuran una nueva estructura social basada en la clase, el género y la raza. Es decir, el proyecto emancipatorio y civilizatorio de la modernidad se configuró a través de lo que considero la "división internacional sexual y racial/colonial del trabajo".

La modernidad no hubiera sido posible sin el ataque sistemático "sobre las condiciones materiales de la reproducción social y contra los principales sujetos de este trabajo, que en la mayor parte de los países son mujeres" (Federici, 20I3, p. I44). En otras palabras, el estudio de las consecuencias de las dimensiones transnacionales del sistema capitalista moderno/colonial permite observar como las desequidades socioeconómicas globales constituyen un mayor impacto sobre las mujeres localizadas en la periferia. Así, las epistemologías del Sur son fundamentales en la búsqueda de un paradigma otro para el análisis de las relaciones económicas neocoloniales Norte-Sur; donde la violencia contra las mujeres juega un papel clave, ejemplo de ello son las zonas de libre comercio, el extractivismo o las guerras.

La deslocalización de las multinacionales a partir de la creación de zonas de libre comercio, que proliferaron durante la década de los setenta, ha permitido abaratar la mano de obra y vulnerar los derechos de la clase trabajadora, produciendo el mayor impacto sobre el trabajo intensivo de la industria textil y electrónica en los países del Sur (Federici, 20I3, p. I I0). Consecuentemente, las mujeres aumentaron enormemente su presencia en el comercio internacional como trabajadoras y configuraron una mano de obra "más hábil y disciplinada", como consecuencia de la dependencia familiar económica que impide que formen parte de organizaciones sindicales u otros procesos para la reivindicación de una mejora de las condiciones laborales y salariales (Quiroga Díaz, 2009, p. 8I). No obstante, esta integración en el mercado internacional no ha eximido a las mujeres del trabajo reproductivo. 
Por este motivo se crearon las zonas de libre comercio, como espacios mercantiles en los países del Sur que han promovido el expolio de sus recursos, además de no favorecer el desarrollo del sector industrial ni las condiciones laborales en los lugares donde se implantan. Los salarios se caracterizan por una gran precarización, incluso por debajo de los mínimos asumidos en los países del Norte, propiciando unas condiciones que han conducido y conducen a la muerte de cientos de mujeres en distintos lugares del planeta desde México a China (Federici, 20 I3, p. I I 2).

Por otro lado, otra forma de violencia contra las mujeres fueron los Programas de Ajuste Estructural (PAE), que impusieron las organizaciones internacionales, como el Banco Mundial y el Fondo Monetario Internacional (FMI), durante la década de los ochenta en países de África y América Latina para fomentar la recuperación económica y el desarrollo capitalista. No obstante, la imposición de los PAE ha sido una estrategia para la destrucción de la agricultura de subsistencia de los países del Sur Global, ya que implica el medio que garantiza la autonomía de su economía local. La imposición de los PAE ha provocado el desplazamiento de mujeres tras el desmantelamiento de sus modos de subsistencia, a través de procesos como la modernización de la agricultura fundamentada en la mercantilización y la exportación. Además, han generado el aumento de recortes en el sector público, sobre todo en sanidad, así como graves consecuencias respecto a la degradación ambiental a partir de la extracción minera, la deforestación y los terrenos vertedero (Federici, 2013, p. I76).

También, la ayuda alimentaria en la que participan los Estados, las organizaciones internacionales y las ONG(D) consiste en un proceso lineal que va desde el ajuste estructural, pasando por la guerra, hasta llegar a la intervención. El trabajo humanitario empezó a cuestionarse con mayor intensidad a partir de la década de los sesenta, como consecuencia del impacto negativo sobre las excolonias que beneficiaba a la economía de guerra. Esta actividad se consolida como un fuerte componente neocolonial y ejemplo de ello fueron los casos de Sudán y Etiopía en los ochenta y Somalia en los noventa, donde la ayuda alimentaria fue instrumentalizada y militarizada.

Finalmente, la guerra se configura como el gran aparato de la máquina neocolonial y que además implica una mayor letalidad sobre las mujeres, de hecho, se habla de un proceso de "feminización de la guerra"|8. La guerra es un medio para la transformación del nuevo orden económico, que se manifiesta en los siguientes fenómenos: (a) el desplazamiento de las poblaciones permite la división de las personas productoras de los medios de producción, (b) la apropiación de las tierras para el uso del mercado global, (c) la mitigación de los movimientos de resistencia que se oponen a estas políticas estructurales y (d) las intervenciones extranjeras, incluso militares. De modo que este fenómeno está estrechamente vinculado con el proceso de globalización, que ha consolidado como ejes de la agenda capitalista un conjunto de políticas que permiten el expolio de los recursos de las excolonias a las metrópolis.

En este sentido, la colonialidad global dio lugar a nuevas relaciones de poder, entre ellas la imposición de una red económica neocolonial basada en la dependencia del Sur al Norte, así como, en el expolio del Norte al Sur. La deslocalización de las multinacionales, la implantación de los PAE por las organizaciones internacionales, el endeudamiento, la ayuda humanitaria o, en el peor de los casos, las guerras, han servido de estrategias para la destrucción de la agricultura de

${ }_{18}$ Para entender el proceso de "feminización de la guerra" y la violencia contra las mujeres, véase la obra de Segato (20I6). 
subsistencia en los países del Sur (Federici, 2013, pp. II5-I48).

A través de la agricultura de subsistencia las mujeres tienen el control de la salud en sus manos, y se consolidan como una fuerte oposición contra la mercantilización de la naturaleza. Las mujeres se convierten en "las agricultoras de subsistencia del planeta", por ejemplo, en el caso africano ellas producen el $80 \%$ de los alimentos para el consumo (Federici, 20I3, p. 25I). No obstante, el fin último es el expolio del territorio y la extracción generalizada de los recursos o "extractivismo económico", que se refiere "al mecanismo que vincula la explotación de recursos naturales y materias primas en la periferia" (Grosfoguel, 20l6b, p. I26).

La histórica extracción de los recursos en la división internacional sexual y racial/colonial del trabajo no solo afecta a la clase trabajadora, entre sus consecuencias incluye "procesos destructivos de la vida y la ecología planetarias" (Grosfoguel, 2016b, p. 127), se convierte en un proceso ecologicida. Para Acosta, "el extractivismo, ha sido un mecanismo de saqueo y apropiación colonial y neocolonial (...) [que] se ha forjado en la explotación de las materias primas indispensables para el desarrollo industrial y el bienestar del Norte global" (20I2, p. 86).

Es decir, la economía global pretende poner fin a todas las formas de economía local que se escapan de la lógica de acumulación y para despojar a las mujeres de sus territorios, tras convertirse en una fuerte amenaza para el desarrollo de la agenda capitalista. En este sentido, es fundamental analizar cómo funciona el patrón de poder de la colonialidad global para entender la subalternización de las vidas; que consiste en un proceso basado en la imposición de las relaciones económicas neocoloniales y, consecuentemente, en el control de los cuerpos de las mujeres, las del Sur Global, las racializadas, las migrantes, las colonizadas y un largo etcétera.

Así, entendemos cómo el sistema económico internacional, tras el proceso colonial y la exportación del capitalismo, ha originado el conflicto capital-vida estableciendo los grupos de vidas vivibles y vidas no vivibles. Es decir, las Relaciones Internacionales juegan un papel "en el empeoramiento de las condiciones de vida de las poblaciones" como consecuencia de las tesis neoliberales, los ideales del libre comercio y el fenómeno de la globalización (Llistar Bosch, 2009, p. 20). Por esta razón, el diálogo hacia una economía feminista descolonial' ${ }^{19}$ deberá partir del análisis de las violencias contra las subalternizadas provocadas por las relaciones económicas neocoloniales.

\section{El diálogo hacia una economía feminista descolonial desde el Norte}

La propuesta dialógica entre la economía feminista y los feminismos descoloniales surge por dos cuestiones principales, por un lado, la clasificación de las personas por "el patrón de poder capitalista" en tres grandes ejes:"trabajo, sexo y raza” (Bidaseca, 2019, p. 19); en segundo lugar, la relación entre la configuración del sistema capitalista moderno/colonial y la imposición global del trabajo reproductivo a las mujeres.

La economía feminista se fundamenta en la crítica a la economía neoclásica, por basarse en un discurso político neoliberal que defiende un sistema teocrático mercantil. Así, la lógica de

\footnotetext{
19 En los trabajos de Quiroga Díaz y Gómez Correal (2013) y Quiroga Díaz (20I4) comienza a desarrollarse el término "economía feminista
} decolonial". 
acumulación se describe en base a tres elementos: androcéntrica, está intrínsicamente ligada a los valores masculinizados; antropocéntrica, defiende la hegemonía de lo humano frente a la naturaleza; y neocolonialista, considera "el capitalismo como un estadio de civilización superior a las economías de subsistencia porque emancipa a las sociedades de los intercambios inmediatos y orgánicos con la naturaleza” (Pérez Orozco, 20I4, p. 206).

Además, desde esta interdisciplina se responsabiliza a la teoría económica ortodoxa de generar la globalización de la división entre lo público y lo privado que dio lugar a la división sexual del trabajo, e ignorar el ámbito local como "el espacio donde la vida se desenvuelve" (Quiroga Díaz, 2009, p. 85). La economía feminista sostiene la necesidad de visibilizar los cuidados introduciéndolos en el análisis macroeconómico, esto implica "un cambio de perspectiva que revela la dependencia de la economía de mercado respecto a la economía del cuidado" (Perea Ozerin, 20l4, p. 73). Por ello, para centrar el análisis económico en la sostenibilidad de la vida es necesario partir del reconocimiento de los cuidados.

En otro orden de cosas, repensar la economía desde los feminismos descoloniales, implica el reconocimiento de las mujeres en la construcción de la historia política y socioeconómica, a partir tanto de las experiencias de las subordinaciones, como de las experiencias de la agencia de las mujeres. De tal forma, se evidencia que el análisis crítico de las relaciones económicas es inseparable del análisis de las relaciones coloniales y raciales. Desde este enfoque, surge el cuestionamiento de la brecha — cada vez mayor — entre el Norte y el Sur, cuya máxima expresión de desigualdad se manifiesta sobre las vidas subalternizadas.

Las epistemologías dominantes han descatalogado otras formas de entender la realidad del mundo, convirtiendo al resto de pueblos que se escapan de la racionalidad civilizatoria en colonizados y barbarizados. Es decir, el colonialismo "va más allá de una práctica de conquista territorial y control político-económico (...) El colonialismo supuso la imposición de formas de acercarse e interpretar el mundo, de maneras de conocer y saber" (Medina Martín, 2012, p. 219). Occidente a través del colonialismo y el capitalismo produjo los saberes y las ciencias sociales dominantes, mientras erradicaba los conocimientos otros.

Por esta razón, los feminismos descoloniales configuran una genealogía feminista que se enfrenta a la colonialidad del ser, del saber y del poder. Así, incorporar esta perspectiva permite cuestionarnos la construcción de Europa y sus relaciones políticas y económicas contemporáneas. La intención es aplicar la perspectiva de una economía feminista descolonial, desde el Norte, hacia la propuesta política de iniciar un proceso de deconstrucción del actual modelo económico, que deberá partir del reconocimiento de la capacidad de agencia de las vidas subalternizadas. El objetivo final es participar en la creación de ese puente Norte-Sur y el andamiaje de redes de conocimientos.

Así mismo, tras analizar cómo el sistema capitalista moderno/colonial se construyó sobre la violencia contra las mujeres, y se sostiene hoy sobre esa misma violencia; una economía feminista descolonial se manifiesta imprescindible para la defensa de la vida en su conjunto planetario. Los consensos que surjan de esta propuesta dialógica serán el hilo para la construcción de una 
alternativa para la que se deberá tener en cuenta los siguientes factores: (a) la ecodependencia ${ }^{20}$ y la necesidad de recurrir al decrecimiento económico, como consecuencia de los límites físicos del planeta y la inminente crisis ecológica; (b) la interdependencia ${ }^{21}$, que comprende que la multidimensionalidad del bien-estar ${ }^{22}$ y la reproducción social deben politizarse y recofingurarse como una corresponsabilidad social; y (c) la redistribución de la riqueza y el fin de la explotación Norte-Sur para acabar con las relaciones neocoloniales del sistema económico internacional.

El reconocimiento de los factores de eco/interdependencia y el fin de las relaciones económicas neocoloniales que generan la brecha Norte-Sur, permitirán avanzar hacia una alternativa basada en un proyecto político que consolide el principio de "los comunes"23. Esta iniciativa surge también de la voluntad de trabajar hacia una economía anticapitalista, gracias a la capacidad de creación de innovadoras formas de reproducción que posibiliten la resistencia a la dependencia del trabajo remunerado y el sometimiento al mercado (Pérez Orozco, 20I4, p. 25I).

Por medio de la idea de los comunes, la alternativa al sistema de muerte debe partir de la defensa del bien-estar global y multidimensional.Además, es imprescindible entender los comunes a partir del reconocimiento de las diferencias y las desigualdades, es decir, deben definirse desde lo local. También la defensa de lo público y las prácticas de otras economías -lejos de la lógica capitalista-; así como, el reconocimiento de los cuidados como un proceso de politización de lo cotidiano y su desnaturalización y desexualización, serán fundamentales para la descentralización de los mercados.

En definitiva, la fase actual y global de translimitación, que ha revelado el desarrollo del sistema capitalista moderno/colonial como ecologicida, manifiesta la necesidad de avanzar hacia una economía feminista descolonial que globalice las vidas vivibles desde lo local. La insostenibilidad del proyecto de la modernidad se traduce en el impacto sobre los pueblos, especialmente sobre las vidas subalternizadas; tras comprobar cómo ha sido creado sobre un proceso de subdesarrollo sistémico. En este sentido, la defensa de la sostenibilidad de la vida se convierte en uno de los consensos fundamentales que se extraen del diálogo entre la economía feminista y los feminismos descoloniales.

\section{Conclusión}

En primer lugar, la dialéctica saber/poder foucaultiana se refleja en el mainstream de las Relaciones Internacionales, que desde los nichos de la academia ha excluido el género, la raza y otras variables coconstitutivas como consecuencia de su lógica eurocéntrica. Así, situar el conocimiento es una

20 La ecodependencia se refiere a la importancia del cuidado de la bioesfera para la sostenibilidad de la vida humana, que parte del reconocimiento de la capacidad limitada del planeta.

21 Pensar la economía desde la interdependencia consiste en comprender la no autosuficiencia de las personas y, por ende, la vulnerabilidad de la vida.

${ }^{22}$ Comprender la economía desde el bien-estar y el mal-estar implica partir de la reflexión sobre cuáles son las vidas vivibles y cuáles no, para saber más véase la obra de Pérez Orozco (20l4).

${ }^{23}$ Para conocer más sobre "los comunes" consúltense las obras de Federici (20I3) y Pérez Orozco (20I4). Además, existen otras alternativas desde los pueblos ancestrales de América Latina como es la concepción del "buen vivir", "vivir bien" o "anaaakuaipa", que se basa en la recuperación de la relación armónica con la naturaleza: “El 'buen vivir' o 'vivir bien' corresponde a una forma de asumir la vida, en la que el respeto y la consideración de las cosas, las personas, los lugares, las experiencias y las prácticas son escenificados para celebrar y ritualizar todo lo que guía la existencia. Con esto se marca una fuerte diferencia con la instrumentalización, la planeación y la estandarización de Occidente, pues este persigue un fin que es alcanzado por unos medios, mientras que con el 'buen vivir' o 'vivir bien' se busca la armonía a partir de integrar lo sagrado, lo terrenal y lo material en una concepción de vida” (Pushaina,Villa y Villa, 2016, p. 248). 
tarea imprescindible desde las Relaciones Internacionales, y solo de esta forma se podrá iniciar un proceso de descolonización y despatriarcalización. El objetivo es promover una ciencia que permita entender la realidad y cómo el sistema opera a escala global, desde las diferencias y los márgenes.

En segundo lugar, sostengo que el reconocimiento global de los cuidados permitirá reclamar que todas las vidas merezcan ser vividas. La necesidad de este proceso surge de la vulnerabilidad, la interdependencia y la ecodependencia de la vida, y pone fin al mito de la autosuficiencia del homo economicus para el capital; contra la privatización de los cuidados en el ámbito de los hogares que se fundamenta en la discriminación de género, sexualidad, raza/etnia y clase social. Es decir, si los procesos económicos terminan en los hogares, estos deben ser incorporados en la esfera pública.

En tercer lugar, se manifiesta como tarea imprescindible la incorporación del diálogo entre los feminismos descoloniales y la economía feminista dentro del análisis de las Relaciones Internacionales. Tras comprobar que el sistema económico internacional sitúa el mercado en el centro, a través de su lógica de acumulación racista/colonial y de la violencia contra las mujeres; la economía feminista descolonial se presenta como una propuesta epistémica —en plena construcción - para acercarnos a una alternativa que centralice la sostenibilidad de la vida.

En último lugar, a lo largo del artículo se ha puesto de manifiesto que la realidad contemporánea de crisis multidimensional -ecológica, ética, política y económica - obliga a cuestionarnos la elección entre un modelo socioeconómico otro o la institucionalización de la barbarie capitalista, heteropatriarcal y racista/colonial. Es decir, urge iniciar un proceso de deconstrucción del sistema económico internacional a favor de la defensa de la sostenibilidad de la vida en su conjunto.

El régimen de colonialidad global, a través de las relaciones neocoloniales económicas ha conseguido la dominación y subordinación de las poblaciones del Sur y los territorios donde estaban/están asentadas. Esta tarea no podría haber concluido sin la ayuda de los Estados-nación occidentales, la expansión del capitalismo y el colonialismo, las organizaciones internacionales, los feminismos hegemónicos y el discurso mainstream de las Relaciones Internacionales.

Las élites occidentales no pusieron fin al colonialismo durante el siglo $X X$, sino que decidieron mantenerlo a través del imaginario político, social y cultural en la división internacional sexual y racial/colonial del trabajo. De este modo, partir de la descolonización de las relaciones económicas es fundamental desde nuestro posicionamiento en el Norte, porque no podremos iniciar una agenda feminista común sin la globalización de la equidad y la justicia social. Es decir, construir una economía feminista descolonial desde Europa deberá partir, en primer lugar, del desvelamiento del sistema de rapiña sobre los países del Sur, así como, señalar la necesidad de iniciar un proceso de redistribución de la riqueza, que además forma parte de una deuda histórica —el centro se ha desarrollado a partir del saqueo y la sobreexplotación de la periferia-.

En síntesis, se manifiesta un proceso insoslayable de deconstrucción del modelo económico actual misógino, colonial, racista, ecologicida y genocida.Ahora más que nunca tenemos el deber de 
crear esa nueva agenda feminista, común, subversiva y global que consiga combatir los nuevos retos que se presentan en la esfera internacional, tales como el ascenso del fascismo y/o la ultraderecha. Necesitamos un discurso otro de las Relaciones Internacionales que haga frente contra aquellos que quieren convertir Europa en un espacio basado en el ecologicidio, la represión, la misoginia, la militarización y las políticas racistas antimigratorias. Por esta razón, la nueva narrativa será feminista y vendrá desde las subalternizadas, las racializadas, las colonizadas, las trans, las migrantes, en definitiva, vendrá desde los márgenes.

\section{Referencias}

Acosta,A. (20I2). Extractivismo y neoextractivismo: dos caras de la misma maldición. En Lang, M., y Mokrani Chávez, D. (Coords.), Más Allá del Desarrollo: Grupo Permanente de Trabajo sobre Alternativas al Desarrollo (pp. 83-I20). Quito.

Barbé, E. y Soriano, J. P. (20I5). Del debate neorrealismo-neoliberalismo a la (re)construcción del discurso dominante en las Relaciones Internacionales. En Del Arenal, C. y Sanahuja, J.A. (Coords), Teoría de las Relaciones Internacionales (pp. 127-I56). Madrid:Tecnos.

Bidaseca, K., Carvajal, F., Mines Cuenya, A., y Núñez Lodwick, L., (20I5). La articulación entre raza, género y clase a partir de Aníbal Quijano. Diálogos interdisciplinarios y lecturas desde el feminismo. Papeles de Trabajo, I0, I8, Pp. 195-218.

Bidaseca, K. (2019). Utopía y estética feminista descolonial: diálogos imaginarios con Aníbal Quijano para la revolución de nuestro tiempo. Revista de Sociología, 28, pp. I5-25

Carrasco, C. (2006). La economía feminista: Una apuesta por otra economía. En María Jesús Vara (Ed.), Estudios sobre género y economía (pp. 29-62). Madrid:Akal.

Castro-Gómez, S. y Grosfoguel, R. (Ed.) (2007). El giro decolonial. Reflexiones para una diversidad epistémica más allá del capitalismo global. Colombia: Siglo del Hombre Editores.

Escobar, A. (2007). La invención del Tercer Mundo. Construcción y deconstrucción del desarrollo. Venezuela: Fundación Editorial el perro y la rana.

Espinosa Miñoso,Y. (2012). De por qué es necesario un feminismo descolonial: diferenciación, dominación co-constitutiva de la modernidad occidental y el fin de la política de identidad. Solar. Revista de Filosofía Iberoamericana, $12(1),|4|-|7|$.

Espinosa Miñoso,Y., Gómez Correal, D., y Ochoa Muñoz, K. (Ed.) (20I4). Tejiendo de otro modo: Feminismo, epistemología y apuestas descoloniales en Abya Yala. Popayán: Editorial Universidad del Cauca.

Federici, S. (20 I0). Calibán y la bruja. Mujeres, cuerpo y acumulación originaria. Madrid:Traficantes de Sueños.

Federici, S. (2013). Revolución en punto cero. Trabajo doméstico, reproducción y luchas feministas. Madrid: Traficantes de Sueños.

Garcés, F. (2007). Las políticas del conocimiento y la colonialidad lingüística y epistémica. En Castro-Gómez, S. y Grosfoguel, R. (Ed.), El giro decolonial. Reflexiones para una diversidad epistémica más allá del capitalismo global (pP. 217-242). Colombia: Siglo del Hombre Editores.

Grosfoguel, R. (2002). Colonial Difference, Geopolitics of Knowledge, and Global Coloniality in the Modern/Colonial Capitalist World-System. Fernand Braudel Center, 25 (3), 203-224.

Grosfoguel, R. (2006). La descolonización de la economía política y los estudios postcoloniales: Transmodernidad, pensamiento fronterizo y colonialidad global. Tabula Rasa, (4), I7-48.

Grosfoguel, R. (20I3). Racismo/sexismo epistémico, universidades occidentalizadas y los cuatro genocidios/epistemicidios del largo siglo XVI. Tabula Rasa, (19), 3I-58.

Grosfoguel, R. (2016a). Caos sistémico, crisis civilizatoria y proyectos descoloniales: Pensar más allá del proceso civilizatorio de la modernidad/colonialidad. Tabula Rasa, (25), I53-I74.

Grosfoguel, R. (2016b). Del “extractivismo económico" al "extractivismo epistémico" y al "extractivismo ontológico": una forma destructiva de conocer, ser y estar en el mundo". Tabula Rasa, (24), I 23-I43.

Llistar Bosch, D. (2009). Anticooperación: Interferencias Norte-Sur. Los problemas del Sur Global no se resuelven con más ayuda internacional. Barcelona: Icaria.

Lugones, M. (2008). Colonialidad y género. Tabula Rasa, 9, 73-I0I.

Mayorquín, N. (08.03.2020). Entrevista a Moira Millán. Recuperado de: http://www.agenciapacourondo.com.ar (2l.03.2020)

edina Martín, R. (20I2). El feminismo postcolonial: Una apuesta crítica para el reconocimiento de las "mujeres colonizadas" como sujetos de cambio y transformación social. En Zurbano Berenguer, B. (Coord.), Mujeres en Oriente Medio. Agentes de desarrollo en un contexto de conflicto (pp. 209-255). Sevilla: Colección Ámbitos para la Comunicación.

Medina Martín, R. (2016). Mujeres Saharauis: Experiencias de Resistencias y Agencias en un de- venir feminista descolonial. 
(Publicada). Universidad Pablo de Olavide. Sevilla.

Medina Martín, R. (2019). Aplicaciones metodológicas en feminismos y de(s)colonialidad. En IV Jornadas de Metodología de Investigación Feminista: violencia y resistencias (Coord.), Otras formas de (des)aprender: investigación feminista en tiempos de violencia, resistencias y decolonialidad (Pp. I I I-I28). País Vasco: Hegoa y SIMReF.

Mendoza, B. (20l4). La epistemología del sur, la colonialidad del género y el feminismo latinoamericano. En Espinosa Miñoso,Y., Gómez Correal, D., y Ochoa Muñoz, K. (Ed.), Tejiendo de otro modo: Feminismo, epistemología y apuestas descoloniales en Abya Yala (pp. 91-103). Popayán: Editorial Universidad del Cauca.

Mignolo, W. (2003). Historias locales/diseños globales. Colonialidad, conocimientos subalternos y pensamiento fronterizo. Madrid: Ediciones Akal.

Mignolo,W. (2005). Cambiando las éticas y las políticas del conocimiento: Lógica de la colonialidad y postcolonialidad imperial. Tabula Rasa, 3, 47-72.

Mignolo, W. (2007). El pensamiento decolonial: desprendimiento y apertura. Un manifiesto. En Castro-Gómez, S. y Grosfoguel, R. (Ed.), El giro decolonial. Reflexiones para una diversidad epistémica más allá del capitalismo global (pp. 25-46). Colombia: Siglo del Hombre Editores.

Moreno Rodríguez,A. (2019). Pensamientos críticos en las Relaciones Internacionales: Herramientas para revisitar la teoría en la disciplina. Relaciones Internacionales, (40), 233-24I.

Ochoa Muñoz, K. (20।4). El debate sobre las y los amerindios: entre el discurso de la bestialización, la feminización y la racialización. En Espinosa Miñoso, Y., Gómez Correal, D., y Ochoa Muñoz, K. (Eds.), Tejiendo de otro modo: Feminismo, epistemología y apuestas descoloniales en Abya Yala (pp. I05-I I 8). Popayán: Editorial Universidad del Cauca.

Perea Ozerin, I. (20I4). El papel del feminismo en el movimiento antiglobalización: contribuciones y desafíos. Revista CIDOB d'Afers Internacionals, I05, 67-88.

Pérez Orozco,A, (20I4). Subversión feminista de la economía.Aportes para un debate sobre el conflicto capital-vida. Madrid: Traficantes de Sueños.

Pushaina, L. E.,Villa, E. y Villa,W. (2016). Referencias cosmogónicas y prácticas del buen vivir según el pensamiento de los wayú de Manaure (La Guajira, Colombia). Tabula Rasa, 24, 243-26I.

Quijano,A. (20I4). Colonialidad del poder, eurocentrismo y América Latina. En CLACSO, Cuestiones y horizontes: de la dependencia histórico-estructural a la colonialidad/descolonialidad del poder (pp. 777-832). Buenos Aires.

Quiroga Díaz, N. (2009). Economías feminista, social y solidaria. Respuestas heterodoxas a la crisis de reproducción en América Latina. Íconos. Revista de Ciencias Sociales, (33), 77-89.

Quiroga Díaz, N. (20I2). Economía del cuidado. Reflexiones para un feminismo decolonial. Revista Casa de la Mujer, $20(2), 97-116$

Quiroga Díaz, N. y Gómez Correal, D. (20I3). ¿Qué tiene para aportar una economía feminista decolonial a las otras economías? América Latina en Movimiento. Recuperado de: https://www.alainet.org/es (I 2.04.2020).

Quiroga Díaz, N. (20I4). Economía feminista y decolonialidad, aportes para la otra economía. Voces en el Fénix, 37, $36-45$.

Ruiz Trejo, M. G.y Betemps, C. (20|4). Epistemologías y prácticas feministas cruzadas: las posibilidades de la traducción y la importancia decolonial. Relaciones Internacionales, (27), 169-179.

Segato, R. L. (20I6). La guerra contra las mujeres. Madrid:Traficantes de Sueños.

Spivak, G. C. (20 I0). Crítica de la razón poscolonial. Hacia una crítica del presente evanescente. Madrid:Akal. 


\section{RELACIONES INTERNACIONALES}

Revista académica cuatrimestral de publicación electrónica Grupo de Estudios de Relaciones Internacionales (GERI)

Universidad Autónoma de Madrid, España

https://revistas.uam.es/relacionesinternacionales

ISSN 1699 - 3950

f facebook.com/RelacionesInternacionales

3. twitter.com/RRInternacional

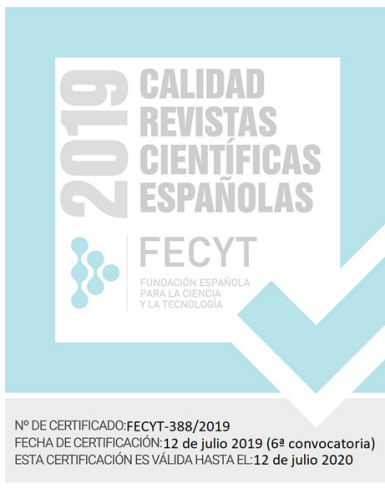

